PREPRINT of an article published in J. Acoust. Soc. Am. 121, 2182-2191 (2007)

\title{
LATERALIZATION DISCRIMINATION OF INTERAURAL TIME \\ DELAYS IN FOUR-PULSE SEQUENCES IN ELECTRIC AND \\ ACOUSTIC HEARING ${ }^{\text {a) }}$
}

\author{
Bernhard Laback ${ }^{\text {b) }}$, Piotr Majdak \\ Acoustics Research Institute, Austrian Academy of Sciences, Reichsratsstrasse 17, \\ A-1010 Vienna, Austria
}

Wolf-Dieter Baumgartner

ENT-Department, Vienna University Hospital, Währinger Gürtel 18-20, A-1097 Vienna, Austria

a) Parts of this work were presented at the 28th Annual Midwinter Research Meeting of the Association for Research in Otolaryngology in February, 2005

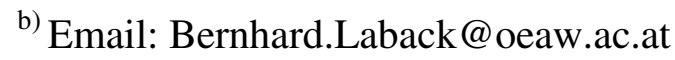

Running title: Interaural time differences in electric hearing

Abbreviated Title: Interaural time differences in electric hearing

Accepted 


\begin{abstract}
This study examined the sensitivity of four CI listeners to ITD in different portions of four-pulse sequences in lateralization discrimination. ITD was present either in all the pulses (referred to as condition Wave), the two middle pulses (Ongoing), the first pulse (Onset), the last pulse (Offset), or both the first and last pulse (Gating). All ITD conditions were tested at different pulse rates $(100,200,400$, and 800 pulses per second, pps). Also, five normal hearing (NH) subjects were tested, listening to an acoustic simulation of CI stimulation. All CI and NH listeners were sensitive in condition Gating at all pulse rates for which they showed sensitivity in condition Wave. The sensitivity in condition Onset increased with the pulse rate for three CI listeners as well as for all NH listeners. The performance in condition Ongoing varied over the subjects. One CI listener showed sensitivity up to $800 \mathrm{pps}$, two up to $400 \mathrm{pps}$, and one at $100 \mathrm{pps}$ only. The group of NH listeners showed sensitivity up to $200 \mathrm{pps}$. The result that CI listeners detect ITD from the middle pulses of short trains indicates the relevance of fine timing of stimulation pulses in lateralization and therefore in CI stimulation strategies.
\end{abstract}

PACS numbers: 43.66.Pn, 43.66.Ts 


\section{INTRODUCTION}

The growing number of bilateral cochlear implantations has raised interest in studies investigating the sensitivity of bilateral cochlear implant (CI) listeners to binaural cues. In particular, the sensitivity to interaural time difference (ITD) in electric hearing has been a subject of interest (see below). ITD is an important cue for the localization of sound sources in the left/right dimension (e.g. Macpherson and Middlebrooks, 2002), for binaural unmasking of speech in noise (e.g. Bronkhorst and Plomp, 1988), and for the perceptual segregation of competing speech sounds (Drennan et al., 2003).

Several psychophysical studies have investigated ITD perception in CI listeners (van Hoesel et al., 1997, 2002, 2003; Lawson et al., 1998; Lawson et al., 2001; Long et al., 2003; Wolford et al., 2003; Laback et al., 2004; Senn et al., 2005; Majdak et al., 2006). They showed that CI listeners are sensitive to ITD, although there is a large interindividual variability in performance. These studies (except for the last three cited) used, besides other stimuli, unmodulated, rectangularly-gated pulse trains as stimuli. The results obtained with this type of stimulus do not reveal to what extent listeners exploit ITD information in the ongoing signal as opposed to the information in the gating portions (onset and offset) of the stimulus. Motivated by those studies, the present study addressed the question if CI listeners are sensitive to ITD information presented either in the ongoing or the gating portions of a pulse train, using a lateralization discrimination task. For that purpose, a specific stimulus was chosen that allowed the strict separation and independent control of ITD in the ongoing and in the gating portions. The stimulus consists of a sequence of four pulses with constant amplitude, in which the first and last pulse represent the gating portions, and the two pulses in the middle represent the 
ongoing portion. In different experimental conditions, ITD information was present either in the ongoing signal, onset, offset, both onset and offset, or in the entire pulse train (to be called waveform ITD). It should be mentioned that while this stimulus has advantages as will be described later, its short duration may complicate the generalization of the outcomes to longer durations.

For an unmodulated electrical pulse train, ongoing ITD is present solely in the fine timing of the individual pulses, which can be referred to as the "fine structure", a term used in the psychoacoustic literature to define the rapidly varying carrier frequency of an acoustic waveform. Thus, in this case, the lateralization discrimination performance for ongoing ITD is a measure of fine structure ITD sensitivity.

It is known from the normal hearing literature that the relative importance of ongoing and gating ITD depends on the rate of the stimulus. Ongoing ITD is the major lateralization cue as long as the frequency of the signal component conveying the ITD, the carrier or the envelope, does not exceed a certain limit. In the case of carrier ITD, the frequency limit is around $1500 \mathrm{~Hz}$ (Klumpp and Eady, 1956; Zwislocki and Feldmann, 1956; Boerger, 1965). In the case of envelope ITD imposed on a high frequency carrier, the frequency limit appears to be lower, depending on the temporal characteristics of the stimulus (Henning, 1974; Hafter and Dye, 1983; Bernstein and Trahiotis, 1994, 2002). ITD in the gating portions, in particular the onset, is more influential at higher signal frequencies (Saberi and Perrott, 1995), particularly in case of ambiguous ongoing ITD cues (Freyman et al., 1997).

To examine these dependencies in electrical hearing, the current study examined lateralization discrimination for each of the ITD conditions described above as a function 
of pulse rate. The measurements obtained from the CI listeners were complemented by measurements on normal hearing $(\mathrm{NH})$ subjects who listened to an acoustic simulation of electric stimulation. Previous studies have shown that some aspects of temporal pitch perception of $\mathrm{NH}$ subjects listening to such an acoustic simulation correspond to the perception of CI listeners (McKay and Carlyon, 1999; Carlyon et al., 2002). Since both temporal pitch perception and ITD perception are based on the temporal properties of the stimulus, the simulation technique used in the cited studies may also mimic some aspects of ITD perception in electric hearing. However, it has to be kept in mind that NH subjects listening to the simulation can discriminate rate pitch up to much higher pulse rates than CI listeners.

Previous studies on NH listeners demonstrated that ITD information in the temporal fine structure is important for the lateralization of sound sources (Wightman and Kistler, 1992; Smith et al., 2002) and for speech perception in noise (Nie et al., 2005; Zeng et al., 2005). If CI listeners were found to be sensitive to ITD in the ongoing signal (and thus the fine structure), then encoding of fine structure cues in future CI stimulation strategies would appear to be a promising approach for improving the ability to lateralize sound sources and to comprehend speech in noise. A better understanding of the particular contribution of ITD information in the ongoing fine structure and in the gating portions as a function of pulse rate could help to improve stimulation strategies for cochlear implants, aiming to maximize the transfer of ITD information. 


\section{METHOD}

\section{A. Subjects and implant system}

Four postlingually deafened CI listeners, implanted bilaterally at the Vienna University Hospital (CI1, CI3, and CI8) and at the University Clinic Würzburg (CI12), were tested. They were selected from a total of eight CI listeners invited for participation in the study. These four listeners fulfilled the selection criterion, as defined by the ability to reproducibly perform left/right discrimination on the basis of $600-\mu \mathrm{s}$ waveform ITD in a sequence of four pulses at a pulse rate of 100 pps. The remaining four listeners showed very low discrimination scores for this baseline condition, even after a full day of training. Table I shows, for all eight listeners invited, the percent correct scores achieved for the baseline condition in a final test at the end of the training. Also included is the performance for a 300-ms version of the same stimulus, to allow comparison with other studies using this stimulus duration. These scores are based on at least 180 item repetitions. Table I also contains data on the patients' etiology. The data of the patients not participating in the experiments are included in the table to make them available for future analysis.

All of the implantees had been supplied with the C40+ system by MED-EL Corp. It generates non-simultaneous biphasic current pulses (cathodic phase first) on up to 12 electrodes (2.4-mm spacing). It provides stimulation in monopolar configuration with an extracochlear ground electrode. The electrodes are numbered in ascending order from apical to basal position in the cochlea.

All four listeners participating in the tests had normal hearing before the onset of deafness. The duration of binaural deafness, i.e., the time period between the beginning 
of deafness at the first ear and the activation of the second CI, was 2 months (CI3), 5.5 months (CI1), 8 years (CI12), and 12 years (CI8). Subject CI3 was supplied with CIs in one operation at both ears. Subjects CI1, CI12, and CI8 were successively supplied with CIs at the two ears with a temporal gap of four months, one year, and two years, respectively. The binaural electric stimulation experience ranged from one month to six years $^{2}$.

Five normal hearing subjects, aged 25-35 years old, participated in this study. None of them had any indication of present or past hearing disorder. Two of the subjects were the first two authors of this study (NH2, NH4). All except one NH subject (NH6) had previous experience with psychoacoustic experiments.

Insert TABLE I about here

\section{B. Apparatus for electric and acoustic stimulation}

A personal computer system was used to control electric and acoustic stimulation. Each implant was controlled by a Research Interface Box (RIB), manufactured at the University of Technology Innsbruck, Austria. The two RIBs were synchronized, providing an interaural accuracy of stimulation timing of $2.5 \mu \mathrm{s}$. Both RIBs were connected to the personal computer system via serial interfaces. Prior to the experiment, the stimuli were verified using a pair of dummy implants (Detektorbox, MED-EL), connected to a two-channel storage oscilloscope (softDSP, SDS 200).

The stimuli for acoustic stimulation were output via a 24-bit stereo A/D-D/A converter (ADDA 2402, Digital Audio Denmark) using a sampling rate of $96 \mathrm{kHz}$ per channel. The converter received the data from the computer via a digital audio interface 
(DIGI 96/8 PRO, RME). The analog signals were sent through a headphone amplifier (HB6, TDT) and an attenuator (PA4, TDT) and presented to the subjects via a circumaural headphone (K501, AKG). Calibration of the headphone signals was performed using a sound level meter (2260, Brüel \& Kjær) connected to an artificial ear (4153, Bruel \& Kjær). The headphone signals were further inspected by digital signal analysis software after digitizing them through the A/D-D/A converter.

\section{Stimuli}

The electric stimuli were trains of four biphasic current pulses with constant amplitude and a phase duration of $26.7 \mu \mathrm{s}$. The current level of the stimuli was adjusted to a comfortable loudness (see Table II). The pulse rate varied between 100 and 800 pps.

The acoustic stimuli used to simulate electric stimulation at a single electrode were similar to those used by McKay and Carlyon (1999) and Carlyon et al. (2002). Monophasic pulses with a duration of $10 \mu \mathrm{s}$ were passed through a band-pass filter with $3 \mathrm{~dB}$ cutoff frequencies at 3900 and $5400 \mathrm{~Hz}$. The filter was designed as a digital eighthorder Butterworth filter, with slopes of $48 \mathrm{~dB} /$ octave. The bandwidth of the filter was broad enough to preserve the modulation in the stimuli. The stimuli were gated with the halves of a Hamming window (duration: $0.6 \mathrm{~ms}$ ) to avoid truncation of the impulse response, which could cause detection of transient cues. The presentation level was set so that a continuously presented pulse train with a pulse rate of $100 \mathrm{pps}$ yielded a rms value of $78 \mathrm{~dB}$ SPL.

The center frequency of the filter $(4590 \mathrm{~Hz})$ was a compromise between two effects. First, the auditory filter bandwidth increases with center frequency; thus, the smearing effect of the auditory filters on the temporal envelope of the stimulus decreases with 
increasing center frequency. This favors the choice of a high frequency. Second, the sensitivity to ITD in amplitude modulated tones decreases for carrier frequencies exceeding 4-6 kHz (Bernstein and Trahiotis, 2002; Henning, 1974). This favors the choice of a low frequency.

The method of simulating electrical stimulation in acoustical hearing by using bandpass filtered pulse trains implies that each pulse corresponds to the impulse response of the band-pass filter used. Thus, each acoustic "pulse" is a complex waveform, having a fine structure and an envelope. Although this differs from electrical pulses which have no fine structure of their own, we consider the band-pass filtered pulse trains as an appropriate simulation of electrical stimulation. This method assumes that information in the envelope of band-pass filtered acoustic pulse trains is analogous to information in the "fine structure" of electric pulse trains, even though the fine structure is not effectively represented in the neural response to high acoustic frequencies.

In acoustical stimulation, interaurally uncorrelated pink noise signals $(50-10050 \mathrm{~Hz})$ were presented continuously at both ears to mask signals outside the desired frequency band. The spectrum level at $4.6 \mathrm{kHz}$ was $15.2 \mathrm{~dB}$ SPL. The noises were generated in realtime and mixed with the pulse trains.

Using a continuous background noise in acoustic stimulation may result in an overall decrease of ITD sensitivity. However, ITD sensitivity measurements with the five NH listeners using 300-ms pulse trains ${ }^{3}$ revealed mean $80 \%$ just noticeable differences (JNDs) of as low as $40 \mu \mathrm{s}$ (SD: $5 \mu \mathrm{s}$ ), which is quite close to the minimum detectable ITD observed in normal hearing. Thus, the noise can have had only a very small effect on ITD sensitivity. 
Insert TABLE II about here

\section{PRETESTS}

Each CI listener completed a series of pretests in order to locate an interaural pair of electrodes eliciting the same pitch percept. These pretests were performed using electric pulse trains with a pulse rate of $100 \mathrm{pps}$ and a duration of $300 \mathrm{~ms}$.

Interaural electrodes with similar pitches seem to be more likely to show ITD sensitivity, although the effect of increasing the interaural place difference can be small (van Hoesel, 2004; Wolford et al., 2003; Long et al., 2003). The procedure to find a pitch-matched electrode pair involved the following steps: a) determination of electric dynamic range and comfortable level for electrodes 1-8 on each ear; b) estimation of monaural pitch sensation across the electrode arrays to determine pitch-matched interaural electrode pair candidates; c) interaural loudness balancing for each interaurally pitch-matched pair candidate; and, d) measurement of pitch discriminability for each interaurally pitch-matched pair candidate and final selection of one pitch-matched pair. More details on the methods can be found in Majdak et al. (2006).

For all CI listeners at least two pitch-matched electrode pairs $(\mathrm{p}<0.01)$ were identified. Table II indicates, for each subject, the electrode pair members finally selected for presenting stimuli in the ITD studies and the corresponding percentage of trials in which the right electrode was judged to be higher in pitch.

The pretests also served to determine comfortable and interaurally loudness-balanced levels of the four-pulse stimuli to be used in the experiments. 


\section{EXPERIMENT I: LATERALIZATION DISCRIMINATION}

This experiment studied the effects of ITD in different signal portions in lateralization discrimination as a function of pulse rate.

\section{A. Procedure}

ITD sensitivity was measured using a "lateralization discrimination" task requiring left/right judgments of a target relative to a comparison stimulus. The first interval contained a comparison stimulus with zero ITD, evoking a centralized auditory image. The second interval contained the target, which differed from the comparison stimulus in that pulses at one ear were delayed relative to the other ear. The subjects were requested to indicate whether the second stimulus was perceived to the left or to the right of the first stimulus by pressing the appropriate button on a response pad. The stimulus intervals were separated by a silent period of $300 \mathrm{~ms}$. Visual indication of the stimulus intervals was provided on a computer screen. Visual feedback about the correctness of the response was provided after each trial. The method of constant stimuli was applied to determine the JND in ITD with respect to lateralization discrimination. Percent correct scores were collected at four to six ITD values to estimate the psychometric function. JNDs were estimated from a maximum-likelihood cumulative Gaussian fit to the percent correct data ${ }^{4}$. The largest ITD presented was $800 \mu$ s and depended on the sensitivity of each subject for the individual conditions. In case of ongoing ITD (the two middle pulses) at the pulse rate of $800 \mathrm{pps}$, however, the largest ITD value was restricted to 500 $\mu \mathrm{s}$, since ongoing ITD approaching half of the interpulse interval can introduce ambiguous cues (Majdak et al., 2006). Each stimulus was presented at least 60 times. In cases where the psychometric function did not exceed 66 percent correct (which occurred 
for some conditions in case of listener CI8), at least 60 further item repetitions were presented in order to reduce the randomness (noise) in the data. A completely randomized design was applied in which all levels of the independent variables and their repetitions were pooled in one "item list" which was then randomized. The subjects took a break after a block of 30 minutes. Depending on the constitution and motivation of the subject, 6 to 10 blocks were completed in one testing day. Before the start of the experiment, the subjects received training using the same procedure as in the main experiment. The training was conducted in three stages. The first stage used a 300-ms version of the baseline condition, the second stage used the baseline condition, and the third stage used a list containing all stimulus conditions of the main experiment. For each stage, the training was continued until the subjects showed stable performance. The four CI listeners fulfilling the criterion for participation required about two hours of training. The other four CI listeners who showed poor performance were trained for one day (6 to 10 blocks) before it was decided to exclude them from this study.

\section{B. Stimulus conditions}

Unmodulated pulse trains, consisting of four pulses, were presented at an interaurally pitch-matched electrode pair, selected in the pretests. The rationale for using a constant number of four pulses at each pulse rate was twofold. First, the pulse amplitude could be held constant across pulse rates, thus avoiding any confounding effects of loudness. It was verified for the $\mathrm{CI}$ listeners and the $\mathrm{NH}$ listeners by an informal loudness estimation task that these stimuli elicit the same loudness at different pulse rates. Second, the number of information units (in terms of pulses) containing ITD remained constant across 
pulse rates, thus avoiding confounding effects of the number of pulses containing ITD information.

Figure 1 illustrates schematically the different types of ITD tested, by showing the amplitude versus time representations of the respective pulse trains at the two ears. The stimulus shown on the top of the figure represents the comparison condition having zero ITD. The stimulus shown beneath, referred to as waveform delay (Wave), contains ITD in each of the interaural pulse pairs. In the condition containing delay in the ongoing signal (Ongoing) the two pulse pairs in the middle of the train contain ITD, whereas the first and last pulse pairs have a zero ITD. In the gating delay condition (Gating) the first and last pulse pairs contain ITD, whereas the two pairs in the middle have zero ITD. The conditions of onset delay (Onset) and offset delay (Offset) contain ITD in the first (onset) pulse pair only and in the last (offset) pulse pair only, respectively. The ITD is always divided between the two ears, i.e., the leading pulse already starts at half the ITD before the reference position at one ear and the lagging pulse starts at half the ITD after the reference position at the opposite ear. This was done to reduce the temporal irregularity, which is a potential monaural discrimination cue.

All ITD types were tested at pulse rates of 100, 200, 400, and 800 pps. Hence, the duration of the stimuli ranged from $40 \mathrm{~ms}$ (at $100 \mathrm{pps)}$ to $5 \mathrm{~ms}$ (at $800 \mathrm{pps}$ ). CI listener CI8 was not tested with the pulse rate of 200 pps.

Insert Fig. 1 about here 


\section{Results}

All subjects reported hearing fused images for all of the conditions tested. Inspection of the distribution of the left/right judgments for each listener revealed sufficient symmetry. Therefore, an adjustment of the percent correct scores to remove response bias was not required. The CI listeners show large inter-individual differences in the overall lateralization discrimination performance. In order to allow the determination of JNDs for all subjects, a threshold criterion of $65 \%$ was used. For the conditions revealing sensitivity at the defined threshold criterion, the psychometric functions are monotonic.

Insert Fig. 2 about here

\section{CI listeners}

Figure 2 shows the JNDs in $\mu$ s for each of the four CI listeners derived from the lateralization discrimination scores, as a function of pulse rate, for the various ITD types. Error bars indicate the $95 \%$ confidence intervals ${ }^{5}$. The significance of the difference between two JNDs was evaluated using a test based on Monte Carlo simulations of the fits to the underlying psychometric functions ${ }^{4}$. Note that some overlap of $95 \%$ confidence intervals for two conditions does not preclude a significant difference between the mean values. JNDs which could not be determined at the specified threshold criterion are marked as ND.

The JNDs for the reference condition Wave increase with the pulse rate in case of listeners CI1 (significant difference between JNDs at 100 and 800 pps: p $=0.003$ ) and 
CI8 (JND at 100 pps: of $398 \mu$ s; JND at 800 pps: undeterminable). In case of CI3 and CI12, the JNDs are approximately constant across pulse rates.

All CI listeners are sensitive to gating ITD at all pulse rates for which sensitivity to waveform ITD was observed. The JNDs are constant across different pulse rates. The apparent decrease of JNDs with increasing rate for listener $\mathrm{CI} 3$ is not statistically significant.

For all CI listeners and at the lowest pulse rate (100 pps), the JNDs for the conditions Ongoing and Gating are larger than those for the condition Wave. These differences are significant for all listeners (largest $p$ value: 0.043), except for CI8. The finding that omission of ITD in either the ongoing or the gating signal portion causes degradation in performance relative to condition Wave implies that both ongoing and gating ITD contribute to lateralization discrimination.

With increasing pulse rate, the CI listeners differ with respect to their sensitivity to ongoing ITD. Listener CI3 shows sensitivity up to 800 pps $^{6}$. Listeners CI8 and CI12 show sensitivity up to 400 pps, and listener CI1 shows sensitivity at 100 pps only.

Three CI listeners (CI1, CI3, and CI12) reveal sensitivity to onset ITD. They also show increasing sensitivity with increasing pulse rate. The significance of this effect for each of the three listeners $\mathrm{CI} 1, \mathrm{CI} 3$, and $\mathrm{CI} 12$ is revealed by the significant differences between the JNDs at 100 and 800 pps ( $\mathrm{p}=0.0001,0.04$, and 0.034 , respectively).

The JNDs for condition Offset are determinable at the pulse rates of 100 and 200 pps only (CI3), at the pulse rate of 100 pps only (CI1 and CI12), and at neither pulse rate (CI8). 
Insert Fig. 3 about here

\section{NH listeners}

The five NH listeners showed homogeneous effects, and therefore Figure 3 shows their mean JNDs. The error bars indicate \pm 1 standard deviation of the mean values across the listeners. The left panel of Figure 3 plots the JNDs determined using the 65\% threshold criterion. For comparison, the right panel shows the JNDs determined for the same data but using the $80 \%$ threshold criterion. Note the different scaling of the ordinates in the two plots. Comparison of JNDs obtained for the two threshold criteria reveals similar effects of the stimulus conditions. This suggests that the choice of the low criterion of $65 \%$ had no major effect on the outcomes.

The significance of differences in JNDs between different test conditions was tested by two-tailed $t$ tests. The performance in condition Wave decreases with the pulse rate. This is reflected by the significantly higher JNDs at $800 \mathrm{pps}$ than at $100 \mathrm{pps}(\mathrm{p}=0.008)$.

The listeners show sensitivity in condition Gating at all pulse rates. The performance is constant across different pulse rates (JNDs at 100 pps vs 800 pps: $\mathrm{p}=0.64$ ).

At the lowest pulse rate (100 pps), the mean JNDs for the conditions Ongoing ( $82 \mu \mathrm{s})$ and Gating $(91 \mu \mathrm{s})$ are higher than those for condition Wave $(48 \mu \mathrm{s})$. The differences Ongoing vs Wave and Gating vs Wave are significant ( $\mathrm{p}=0.01$ and 0.001 , respectively). In addition, the mean JNDs for conditions Onset $(220 \mu \mathrm{s})$ and Offset $(193 \mu \mathrm{s})$ are significantly higher than those for condition Gating $(91 \mu \mathrm{s})(\mathrm{p}=0.02$ and 0.01 , respectively). Taken together, the results at $100 \mathrm{pps}$ indicate perceptual contribution of each interaural pulse pair. 
With increasing pulse rate, the sensitivity to ongoing ITD decreases monotonically. The highest rate showing determinable JNDs is 200 pps in four listeners and 400 pps in one listener. The JND determinable for one listener at 400 pps amounts to $486 \mu$ s.

The sensitivity to onset ITD increases monotonically with the pulse rate. The JNDs at 100 and 800 pps differ significantly from each other $(\mathrm{p}=0.007)$. At $800 \mathrm{pps}$, the JND in

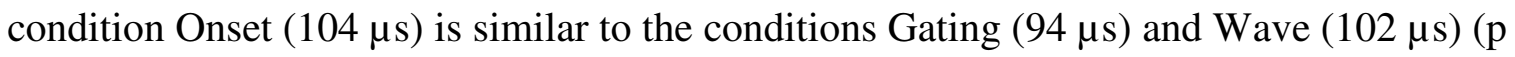
$=0.22$ and 0.84 , respectively). In contrast, the sensitivity to offset ITD decreases with the pulse rate. At 400 pps, the JNDs for condition Offset are high for two of the listeners (mean value: $547 \mu \mathrm{s}$ ) and undeterminable for the other three listeners. Figure 3 shows the mean JND of the two listeners for whom a JND could be determined. At $800 \mathrm{pps}$, they are undeterminable for all listeners. Comparing the JNDs for conditions Onset and Offset with those for condition Gating reveals complementary contributions of onset and offset ITD as a function of pulse rate.

\section{Discussion}

\section{CI listeners}

Three main effects can be observed from the data collected in Experiment I with the four CI listeners, despite considerable inter-individual differences. First, gating ITD contributes to lateralization discrimination at all pulse rates for which sensitivity to waveform ITD was observed. Second, the three CI listeners revealing sensitivity to onset ITD show increasing contribution of onset ITD with increasing pulse rate. Third, and most importantly, all subjects show sensitivity to ITD in the two pulses in the middle of the train, the condition referred to as Ongoing: one listener up to $800 \mathrm{pps}$, two listeners up to $400 \mathrm{pps}$, and one listener at $100 \mathrm{pps}$ only. Sensitivity in condition Ongoing implies that 
ITD in the temporal fine structure of the short pulse trains contributes to lateralization discrimination. These stimuli present "pure" fine structure ITD, containing no other ITD cues in the onset, offset, or ongoing envelope. Majdak et al. (2006) studied fine structure ITD sensitivity in four CI listeners using amplitude modulated pulse trains with a 300-ms duration. In that study, fine structure ITD was created by delaying the pulses at one ear and subsequently applying a trapezoidal envelope with zero ITD. This could potentially involve confounding effects of conflicting ITD cues at the onset. Namely, the first audible pulse could be that on the lagging side, since the first pulse on the leading side was at the absolute threshold. However, the finding of high fine-structure-ITD sensitivity up to $800 \mathrm{pps}$ in that study indicates that conflicting ITD cues during the onset played a minor role.

The conditions Ongoing and Wave at 800 pps may have introduced ambiguous cues for ITD values approaching half of the interpulse interval. This would be reflected by a decline of the psychometric function in that ITD range. In fact, these psychometric functions showed no such decline at the largest ITDs tested, which were $500 \mu$ s and 600 $\mu$ s in conditions Ongoing and Wave, respectively. Thus, it is unlikely that ambiguity had a strong influence. This is in contrast to the results of Majdak et al. (2006) who observed a pronounced decline of the psychometric functions for ITDs between approximately one quarter and one half of the inter-pulse period, using 300-ms pulse trains with ITD in the fine structure only. The absence of non-monotonicity of the psychometric functions in the current study could be due to the short duration of the stimuli, assuming that for short stimuli the onset is more dominant in resolving the ambiguity than for longer stimuli. 
For the listeners $\mathrm{CI} 1, \mathrm{CI} 3$, and $\mathrm{CI} 12$, the sensitivity to gating ITD is qualitatively consistent at the different pulse rates with the corresponding contributions of onset and offset ITD. This leads to the assumption that in the condition Gating the contributions of onset and offset ITD are combined. For CI8, however, gating ITD was found to contribute despite undeterminable JNDs in conditions Onset and Offset. This may indicate that either onset or offset ITD alone are too weak to be evaluated.

\section{NH listeners}

For the NH listeners, the decrease in sensitivity in condition Ongoing with increasing pulse rate is qualitatively consistent with studies from the NH literature. Hafter and Dye (1983) and Saberi (1996) investigated the effect of pulse rate on the perceptual contribution of the ongoing signal using band-pass filtered clicks. These studies observed decreasing contribution of ITD in the clicks following the onset for rates exceeding 200 clicks per second. The maximum pulse rate where all $\mathrm{NH}$ listeners of the current study were sensitive to ITD in the two pulses in the middle of the train (condition Ongoing ITD) was 200 pps. This is qualitatively comparable with the results of a study by Bernstein and Trahiotis (2002). They found sensitivity to ongoing ITD for modulation frequencies up to $256 \mathrm{~Hz}$, using sinusoidally amplitude-modulated and "transposed" 4$\mathrm{kHz}$ tones.

The high sensitivity to onset ITD at $400 \mathrm{pps}$ and the complete dominance of onset ITD observed at $800 \mathrm{pps}$ in the current study is qualitatively consistent with the results of Saberi and Perrott (1995) and Freyman et al. (1997). They showed that click trains with rates equal to or greater than 500 pps are lateralized toward the ear favored by ITD in the 
onset click even if the remaining clicks in the stimulus have an ITD favoring the opposite ear.

The performance in condition Gating was found to be constant across different pulse rates. This seems to be an effect of the complementary contributions of onset and offset ITD as a function of pulse rate, as has also been observed for three of the CI listeners.

\section{EXPERIMENT II: MONAURAL DETECTION}

This experiment was intended to verify that the lateralization judgments obtained in Experiment I were based on binaural information rather than on monaural cues such as periodicity pitch or timbre. Such cues could theoretically have been exploited by the listeners in case of all ITD types besides Wave. Condition Ongoing, for example, involves a change in the interpulse interval from the first to the second and from the second to the third (and last) interpulse interval. The experiment tested if the subjects exceed chance performance in detecting monaural versions of the stimuli used in Experiment I. If the subjects do not exceed chance performance then the performance in Experiment I was not based on monaural cues. In case the subjects exceed chance performance, the data obtained in Experiment I could have been based on monaural cues.

\section{A. Method}

A three-interval, two-alternative forced-choice procedure (oddity task) was used in which the listener had to judge which stimulus was different from the other two. Visual feedback about the correctness of the response was provided after each trial. The "odd" stimulus was a monaural version of the target stimulus used for lateralization discrimination (having an irregular interpulse interval). The comparison stimulus was a 
monaural version of the respective reference stimulus (having a regular interpulse interval). The magnitude of the deviation from the regular interpulse interval, chosen for each condition, corresponded to half the value of the ITD tested in Experiment I which was just above the lateralization JND. All combinations of pulse rates and ITD types (except for Wave) of Experiment I were tested. A total of 36 stimulus presentations were applied for each condition. The stimuli were presented in completely randomized order in a single test session. A training phase with 20 presentations of each stimulus condition was completed before collecting data. All other aspects of the method and stimuli were identical as in Experiment I.

\section{B. Results}

The highest performance achieved by each of the listeners for all of the conditions was: CI1: 47.2\%, CI3: 39\%, CI8: 44.4\%, NH2: 47.2\%, NH3: 47.2\%, NH4: 36\%, NH5: 42\%, NH6: $47.2 \%$. In all cases the performance fell within the range of chance rating (p $>0.05)$. Thus, it was concluded that the lateralization discrimination thresholds obtained in Experiment I were entirely based on binaural cues.

\section{GENERAL DISCUSSION AND CONCLUSIONS}

This study investigated the contribution of ITD in various portions of short unmodulated sequences of four pulses in lateralization discrimination as a function of pulse rate. All four cochlear implant listeners were sensitive, at least at low rates, to ITD in the signal portion referred to as ongoing signal, consisting of the two pulses in the middle of the train. Thus, they were sensitive to ITD in the temporal fine structure of the pulse sequences. However, the listeners differed greatly with respect to the highest pulse rate 
showing sensitivity (100 pps in one listener, $400 \mathrm{pps}$ in two listeners, and $800 \mathrm{pps}$ in the fourth listener). Furthermore, all listeners were found to be sensitive to ITD in the gating pulses at all pulse rates for which they showed sensitivity to waveform ITD. For the three listeners revealing sensitivity to onset ITD, its contribution was shown to increase with the pulse rate.

Because only four CI listeners participated in the experiments, no general conclusions can be drawn for the population of bilateral CI listeners. The data should rather be considered as case studies. As already mentioned, four of the eight CI listeners invited for participation in the tests showed low and unstable sensitivity for a baseline condition and were therefore not included for further participation in the experiments.

The stimuli were designed to avoid the influence of confounding parameters such as differences in the number of pulses and the amplitude in the comparison across different pulse rates. By using the same number of four pulses and a constant amplitude, the same information units were presented at each pulse rate, which facilitates the comparison across pulse rates.

It is also important to keep in mind that the four-pulse sequences used in this study are quite short, in particular at the higher pulse rates. It is possible that for the higher rates the neurons were in refractory state immediately after the onset pulse and thus did not respond to the two pulses in the middle presenting "ongoing ITD". In everyday situations, sustained timing cues are likely to continue for more than just two electrical pulses, and thus the weighting of different types of ITD cues may differ.

To check the outcomes for longer stimuli with a constant duration across pulse rates, additional data have been collected. The stimuli had the same duration of $300 \mathrm{~ms}$ at all 
pulse rates $(100,400$, and $800 \mathrm{pps})$. The stimulus amplitude at $100 \mathrm{pps}$ was the same as in Experiment I and for higher pulse rates it was adjusted to elicit equal loudness. The conditions Wave, Ongoing, and Gating were tested. The methodology was the same as in Experiment I. Since this experiment was done with just one CI listener (CI3, who was available for further testing), the interpretation of the results has to be considered as preliminary. The results confirmed two main findings from Experiment I. First, the listener was able to lateralize upon ongoing ITD. Second, gating ITD contributed to lateralization discrimination at all three pulse rates tested. Furthermore, up to $400 \mathrm{pps}$, the sensitivity to ongoing ITD was higher for the 300-ms stimuli than for the four-pulse stimuli. This improvement is most likely due to temporal integration of ITD information. At 800 pps, however, no sensitivity to ongoing ITD was observed, which is in contrast to the results for the four-pulse stimuli. This may be related to the lower amplitude compared to the four-pulse stimuli. Lowering of the amplitude was necessary to obtain equal loudness increasing the stimulus duration. In summary, these results reveal an interaction between the effects of the parameter pulse rate and the parameters pulse number and amplitude. To use a constant number of information units containing ITD at each pulse rate in Experiment I circumvented these interactions.

Individual differences in the upper rate limit for sensitivity in condition Ongoing, as observed in Experiment I, suggest that some unknown factors besides those controlled in the experiments (interaural pitch and loudness matching) can limit the perception of ITD in the two pulses following the onset in electric hearing. One potential factor could be the decay of internal excitation caused by pulsatile electric stimulation. Different studies have shown that the recovery from forward masking, which may be related to the decay 
of excitation, can vary considerably between CI listeners (Chatterjee, 1999; Nelson and Donaldson, 2002).

One of the CI listeners showed sensitivity in condition Ongoing up to $800 \mathrm{pps}$, whereas four out of five NH listeners showed an upper rate limit of $200 \mathrm{pps}$. This difference may be related to the specific properties of electric and acoustic stimulation. First, a limiting factor in acoustic hearing may be the critical band filtering on the basilar membrane, which is bypassed in electric hearing. Ringing of the auditory filters in acoustic stimulation effectively reduces the modulation depth. This could make it difficult to extract ITD information from the pulses following the onset at higher pulse rates $^{7}$. Second, phase locking is known to be stronger in electric hearing than in acoustic hearing due to bypassing the synaptic mechanism at the hair cell (Abbas, 1993).

Experiment II verified that monaural cues had no influence on the lateralization discrimination scores obtained in Experiment I. It was concluded that the performance in Experiment I was entirely based on binaural cues.

The finding that temporal fine structure cues can be exploited in electric hearing for pulse rates as high as $800 \mathrm{pps}$ is supported by a recent study on monaural rate discrimination by Chen and Zeng (2004), who reported the ability of three CI listeners to detect sinusoidal frequency modulation at rates of the standard stimulus up to 1000 pps. This finding differs from previous studies, which have shown that CI subjects typically cannot detect a pitch difference based on rate above 300-500 pps of the standard stimulus (e.g. Zeng, 2002). A recent study performed by the authors of the present study (Majdak et al., 2006) investigated the effects of ITD in the fine structure, using amplitudemodulated pulse trains. As in the current study, large differences between individual CI 
listeners were observed with respect to the highest pulse rate showing effects of fine structure ITD. Van Hoesel and Tyler (2003) and van Hoesel (2004) presented, for the first time, performance measures of CI listeners tested with a new stimulation strategy designed to encode fine structure ITD cues. No clear advantage in sound source localization could be observed for the new strategy compared to conventional strategies, which discard fine structure information. More work is needed to determine the potentials of stimulation strategies encoding ITD information in the fine structure, considering the complexity of the parameters and effects involved. For example, channel interactions due to current spread may disrupt low-frequency ITD cues in the fine structure. The practical conclusion from the data collected in this study is that bilateral CI listeners may benefit from encoding fine structure ITD information in future CI stimulation strategies with respect to the localization of sound sources in the left/right dimension.

\section{SUMMARY}

Four bilateral cochlear implant listeners were tested on their ability in left/right discrimination on the basis of ITD in different portions of four-pulse sequences, as a function of pulse rate. ITD information was presented in the two middle pulses, in the gating portions (onset and offset pulses), or in the entire train. Furthermore, five normal hearing subjects were tested, listening to simulations of electrical stimulation.

(1) One of the CI listeners showed sensitivity to ITD in the two middle pulses up to 800 pps, two CI listeners up to 400 pps, and one CI listener up to 100 pps. Four NH listeners showed sensitivity up to $200 \mathrm{pps}$, one up to $400 \mathrm{pps}$. 
(2) For all $\mathrm{CI}$ and NH listeners, gating ITD contributed at all pulse rates. The sensitivity to onset ITD increased with the pulse rate for three CI listeners as well as for all $\mathrm{NH}$ listeners.

(3) A monaural detection experiment verified that the listeners did not make use of monaural cues when performing the lateralization discrimination task.

\section{ACKNOWLEDGMENTS}

We are indebted to our test persons, in particular the CI listeners, for their patience while performing the longsome tests. Steve Colburn and an anonymous reviewer provided valuable comments on a previous version of this article. We are grateful to Steve Greenberg and Christopher Long for fruitful discussions and to Matthew Goupell and Brian Gygi for helpful comments on an earlier version of this paper. We thank MEDEL Corporation for providing the equipment for direct electric stimulation. This study was supported by the Austrian Academy of Sciences.

\section{ENDNOTES}

\footnotetext{
${ }^{1}$ Referring to the "fine structure" of an electrical pulse train without envelope modulation is uncommon, but appears appropriate in this context.

${ }^{2}$ The short binaural experience of CI listeners CI3 and CI8 may be considered as a potential shortcoming. We had the opportunity to perform repeated tests with these two listeners two years and one year, respectively, after the main tests and observed no change in ITD sensitivity (see also Majdak et al., 2006). It should be considered that clinical CI systems which use constant pulse rates and thus discard fine binaural timing
} 
cues provide no stable fine structure ITD cues in everyday listening. This supports our opinion that the short binaural CI experience of the listeners CI3 and CI8 did not negatively influence ITD their sensitivity.

${ }^{3}$ Applying waveform ITD in unmodulated pulse trains with 100 pps and using the same methods as in experiment I.

${ }^{4}$ Using psignifit version 2.5.41 (see http://bootstrap-software.org/psignifit/), a software package for fitting psychometric functions to psychophysical data, described in Wichmann and Hill (2001a) and Wichmann and Hill (2001b).

${ }^{5}$ Confidence intervals were found by the $\mathrm{BC}_{\mathrm{a}}$ bootstrap method implemented by psignifit, based on 1999 simulations (see Wichmann and Hill, 2001b).

${ }^{6}$ Since the observation of sensitivity up to 800 pps was unexpected, the measurements for condition Ongoing at 100, 400, and 800 pps were repeated at another testing day revealing exactly the same results.

${ }^{7}$ The JNDs obtained for the NH listeners might depend on the frequency region of the bandpass filter applied on the stimulus and therefore be somewhat arbitrary. Assuming that ringing of the auditory filters is the limiting factor, lower JNDs would be expected at higher frequency regions where the impulse responses of the auditory filters are shorter. On the other hand, Bernstein and Trahiotis (2002) found that the upper rate limit in the sensitivity to ongoing ITD does not increase with the center frequency for center frequencies from 4 to $10 \mathrm{kHz}$, thus not supporting the assumption that cochlear filtering is the limiting factor. However, the stimuli used by Bernstein and Trahiotis (2002) had a constant bandwidth in $\mathrm{Hz}$, thus the bandwidth in ERB decreased with the center freqency. It may be that using a constant bandwidth in the ERB scale would favor the performance 
at higher center frequencies relative to that at the lower frequencies because of the larger number of stimulated neurons and/or because of the larger modulation depth at the output of the cochlear filter due to the larger bandwidth. A study attempting to clarify these issues with normal hearing listeners is currently underway.

\section{REFERENCES}

Abbas, P. J. (1993). “Electrophysiology,” in Cochlear Implants: Audiological Foundations, edited by R. S. Tyler (Singular Publishing Group, Inc.)

Bernstein, L. R., and Trahiotis, C. (1994). "Detection of interaural delay in highfrequency sinusoidally amplitude-modulated tones, two-tone complexes, and bands of noise," J. Acoust. Soc. Am. 95, 3561-3567.

Bernstein, L. R., and Trahiotis, C. (2002). "Enhancing sensitivity to interaural delays at high frequencies by using "transposed stimuli"," J. Acoust. Soc. Am. 112 , 1026-1036. Boerger, G. (1965). "The localization of Gaussian tones," unpublished dissertation, Technical University of Berlin.

Bronkhorst, A. W., and Plomp, R. (1988). "The effect of head-induced interaural time and level differences on speech intelligibility in noise," J. Acoust. Soc. Am. 83, 15081516.

Chatterjee, M. (1999). “Temporal mechanisms underlying recovery from forward masking in multielectrode-implant listeners,” J. Acoust. Soc. Am. 105, 1853-1863. Chen, H., and Zeng, F.G. (2004). "Frequency modulation detection in cochlear implant subjects,” J. Acoust. Soc. Am. 116, 2269-2277. 
Carlyon, R. P., van Wieringen, A., Long, C. J., Deeks, J. M., Wouters, J. (2002).

“Temporal pitch mechanisms in acoustic and electric hearing,” J. Acoust. Soc. A. 112, 621-633.

Collins, L. M., Zwolan, T.A., Wakefield, G.H. (1997). "Comparison of electrode discrimination, pitch ranking, and pitch scaling data in postlingually deafened adult cochlear implant subjects,” J. Acoust. Soc. Am. 101, 440-455.

Drennan, W. R., Gatehouse, S., and Lever, C. (2003). "Perceptual segregation of competing speech sounds: the role of spatial location,” J. Acoust. Soc. Am. 114, 21782189.

Freyman, R. L., Zurek, P. M., Balakrishnan, U., and Chiang, Y. C. (1997). “Onset dominance in lateralization,” J. Acoust. Soc. Am. 101, 1649-1659.

Hafter, E. R., and Dye, R. H. Jr. (1983). "Detection of interaural differences of time in trains of high-frequency clicks as a function of interclick interval and number," J. Acoust. Soc. Am. 73, 644-651.

Henning, G. B. (1974). "Detectability of interaural delay in high-frequency complex waveforms," J. Acoust. Soc. Am. 55, 84-90.

Klumpp, R. G., and Eady, H. R. (1956). "Some measurements of interaural time difference thresholds," J. Acoust. Soc. Am. 28, 859-860.

Laback, B., Pok, S. M., Baumgartner, W. D., Deutsch, W. A., and Schmid, K. (2004). "Sensitivity to interaural level and envelope time diffenences of two bilateral cochlear implant listeners using clinical sound processors," Ear Hear. 25, 5, 488-500. 
Lawson, D. T., Wilson, B. S., Zerbi, M., van den Honert, C., Finley, C. C., Farmer, J. C. Jr., McElveen, J. T. Jr., and Roush, P. A. (1998). "Bilateral cochlear implants controlled by a single speech processor," American Journal of Otology. 19, 758-761.

Lawson, D. T., Wolford, R., Brill, S., Schatzer, R., and Wilson, B. S. (2001). Twelfth quarterly progress report: Speech processors for auditory prostheses, Center of Auditory Prosthesis Research, Research Triangle Institute, NIH project N01-DC-8-2105. Bethesda. http://www.nidcd.nih.gov/staticresources/funding/programs/npp/pdf/archived/N01-DC8-2105QPR12.pdf

(last viewed on Nov. 10th 2006)

Long, C. J., Eddington, D. K., Colburn, H. S., and Rabinowitz, W. M. (2003). "Binaural sensitivity as a function of interaural electrode position with a bilateral cochlear implant user,” J. Acoust. Soc. Am. 114, 1565-1574.

Majdak, P., Laback, B., and Baumgartner, W. D. (2006). "Effects of interaural time differences in fine structure and envelope on lateral discrimination in electric hearing," J. Acoust. Soc. Am. 120, 2190-2201.

Macpherson, E. A., and Middlebrooks, J. C. (2002). "Listener weighting of cues for lateral angle: the duplex theory of sound localization revisited," J. Acoust. Soc. Am. $111,2219-3622$.

McKay, C. M., and Carlyon, R. P. (1999). "Dual temporal pitch percepts from acoustic and electric amplitude-modulated pulse trains," J. Acoust. Soc. Am. 105, 347-357. Nelson, D. A., and Donaldson, G. S. (2002). "Psychophysical recovery from pulse-train forward masking in electric hearing,” J. Acoust. Soc. Am. 112, 2932-2947. 
Nie, K., Stickney, G., Zeng, F. G. (2005). "Encoding frequency modulation to improve cochlear implant performance in noise,” IEEE Trans. Biomed. Eng. 52, 64-73.

Puckette, M. (1996). "Pure Data: another integrated computer music environment," proceedings of second intercollege computer music concerts, Tachikawa, Japan, 37-41. Saberi, K. and Perrott, D. R. (1995). "Lateralization of click-trains with opposing onset and ongoing interaural delays," Acustica. 81, 272-275.

Saberi, K. (1996). "Observer weighting of interaural delays in filtered impulses," Perception and Psychophysics. 58, 1037-1046.

Senn, P., Kompis, M., Vischer, M., and Haeusler, R. (2005). "Minimum audible angle, just noticeable interaural differences and speech intelligibility with bilateral cochlear implants using clinical speech processors," Audiology Neurootology 10, 342-352. Smith, Z. M., Oxenham, A. O., and Delgutte, B. (2002). "Chimaeric sounds reveal dichotomies in auditory perception," Nature 416, 87-90.

van Hoesel, R. J., and Clark, G. M. (1997). "Psychophysical studies with two binaural cochlear implant subjects," J. Acoust. Soc. Am. 102, 495-507.

van Hoesel, R. J., Ramsden, R., and Odriscoll, M. (2002). "Sound-direction identification, interaural time delay discrimination, and speech intelligibility advantages in noise for a bilateral cochlear implant user," Ear Hear. 23, (2):137-149.

van Hoesel, R. J., and Tyler, R. S. (2003). "Speech perception, localization, and lateralization with bilateral cochlear implants," J. Acoust. Soc. Am. 113, 1617-1630. van Hoesel, R. J. (2004). "Exploring the benefits of bilateral cochlear implants," Audiology Neurootology 9, 234-246. 
Wichmann, F. A., and Hill, N. J. (2001a). “The psychometric function: I. Fitting, sampling, and goodness of fit," Perception and Psychophysics, 63, 1293-1313.

Wichmann, F. A. \& Hill, N. J. (2001b). “The psychometric function: II. Bootstrap-based confidence intervals and sampling," Perception and Psychophysics. 63, 1314-1329.

Wightman, F. L., and Kistler, D. J. (1992). “The dominant role of low-frequency interaural time differences in sound localization,” J. Acoust. Soc. Am. 91, 1648-1661.

Wolford, R., Lawson, D. T., Schatzer, R., Xiaoan, S., and Wilson, B. S. (2003). Fourth quarterly progress report: Speech processors for auditory prostheses, Center of Auditory Prosthesis Research, Research Triangle Institute, NIH project N01-DC-2-1002. Bethesda. http://www.nidcd.nih.gov/staticresources/funding/programs/npp/pdf/N01-DC-21002QPR04.pdf (last viewed on Nov. 10th 2006)

Zeng, F. G. (2002). “Temporal pitch in electric hearing,” Hear. Res. 174, 101-106. Zeng, F. G., Nie, K., Stickney, G. S., Kong, Y. Y., Vongphoe, M., Bhargave, A., Wei, C., Cao, K. (2005). "Speech recognition with amplitude and frequency modulations," Proc. Natl. Acad. Sci. U S A. 102, 2293-2298.

Zwislocki, J., and Feldman, R. S. (1956). "Just noticeable differences in dichotic phase ," J. Acoust. Soc. Am. 28, 860-864. 
TABLE I. Etiology of the four CI listeners completing the experiments (CI1, CI3, CI8, and CI12). Also included are the data of the four CI listeners who showed too poor sensitivity in the baseline condition (four pulses; $100 \mathrm{pps}$; waveform ITD of $600 \mu \mathrm{s}$ ) and thus were not included for participation in the experiments. The rightmost columns show the lateralization discrimination scores for the baseline condition and the 300-ms version of the baseline condition.

\begin{tabular}{|c|c|c|c|c|c|c|c|c|c|c|}
\hline \multirow[t]{2}{*}{$\overline{\text { Subject }}$} & \multirow{2}{*}{$\begin{array}{l}\begin{array}{l}\text { Participating in } \\
\text { the experiments }\end{array} \\
\end{array}$} & \multirow[t]{2}{*}{$\overline{\overline{E \text { Etiology }}}$} & \multirow{2}{*}{$\begin{array}{l}\text { Age } \\
(\mathrm{yr})\end{array}$} & \multicolumn{2}{|c|}{ Age at implantation (yr) } & \multicolumn{2}{|c|}{ Duration of deafness } & \multirow{2}{*}{$\begin{array}{c}\text { Binaural electrical } \\
\text { stimulation experience }\end{array}$} & \multirow{2}{*}{$\begin{array}{c}\text { Performance for baseline } \\
\text { condition (in \% correct) }\end{array}$} & \multirow{2}{*}{$\begin{array}{l}\text { Performance for } 300 \mathrm{~ms} \text { version } \\
\text { of baseline condition (in \% correct) }\end{array}$} \\
\hline & & & & $\mathrm{L}$ & $\mathbf{R}$ & $\mathrm{L}$ & $\mathbf{R}$ & & & \\
\hline Cl1 & yes & Meningitis & 20 & 14 & 14 & $5.5 \mathrm{mo}$ & $1.5 \mathrm{mo}$ & $6 \mathrm{yr}$ & 80.0 & 98.3 \\
\hline $\mathrm{Cl} 3$ & yes & Meningitis & 21 & 21 & 21 & $2 \mathrm{mo}$ & $2 \mathrm{mo}$ & $1 \mathrm{mo}$ & 96.0 & 99.0 \\
\hline $\mathrm{Cl} 8$ & yes & Osteogenesis imperfekta & 41 & 41 & 39 & $3 \mathrm{yr}$ & $12 \mathrm{yr}$ & $2 \mathrm{mo}$ & 73.0 & 77.0 \\
\hline $\mathrm{Cl} 12$ & yes & Sudden hearing loss & 40 & 35 & 34 & $8 \mathrm{yr}$ & $3 \mathrm{yr}$ & $5 \mathrm{yr}$ & 95.0 & 99.0 \\
\hline $\mathrm{Cl} 2$ & no & Skull trauma & 58 & 54 & 48 & $21 \mathrm{yr}$ & $25 \mathrm{yr}$ & $4 \mathrm{yr}$ & 58.3 & 70.0 \\
\hline $\mathrm{Cl} 16$ & no & Progressive & 42 & 41 & 39 & $8 \mathrm{yr}$ & $8 \mathrm{yr}$ & $1 \mathrm{yr}$ & 60.0 & 65.0 \\
\hline $\mathrm{Cl} 15$ & no & Otosclerosis & 44 & 35 & 42 & $2 \mathrm{yr}$ & $9 \mathrm{yr}$ & $2 \mathrm{yr}$ & 59.4 & 73.7 \\
\hline $\mathrm{Cl9}$ & no & Progressive & 58 & 50 & 51 & $5 \mathrm{yr}$ & $5 \mathrm{yr}$ & $7 \mathrm{yr}$ & 60.6 & 75.9 \\
\hline
\end{tabular}


TABLE II. Electric stimulation parameters. The electrodes are numbered from apex to base in ascending order. With respect to the parameter "right electrode higher" in percent, any value $\leq 24$ or $\geq 76$ indicates significant pitch discriminability $(\mathrm{p}<0.01)$. The stimulation levels are specified in $\mu \mathrm{A}$.

\begin{tabular}{|c|c|c|c|}
\hline \multirow[t]{2}{*}{ Subject } & $\begin{array}{c}\text { Test } \\
\text { electrodes }\end{array}$ & \multirow{2}{*}{$\begin{array}{c}\text { Right } \\
\text { electrode higher } \\
\text { (in \%) }\end{array}$} & \multirow{2}{*}{$\begin{array}{l}\begin{array}{c}\text { Current levels (in } \mu \mathrm{A}) \\
\text { used in exp. I and II }\end{array} \\
\mathrm{L} / \mathrm{R}\end{array}$} \\
\hline & $L / R$ & & \\
\hline $\mathrm{Cl} 1$ & $4 / 1$ & 50.0 & $261 / 248$ \\
\hline $\mathrm{Cl} 3$ & $4 / 3$ & 42.3 & 265 / 283 \\
\hline $\mathrm{Cl} 8$ & $7 / 5$ & 45.0 & 376 / 358 \\
\hline $\mathrm{Cl} 12$ & $2 / 2$ & 53.8 & 547 / 601 \\
\hline
\end{tabular}




\section{FIGURE CAPTIONS}

Fig. 1 (color online). Schematic illustration of the different ITD types: The amplitude vs time representations of the pulse trains at the two ears are shown. Note that electric pulses were actually biphasic and acoustic pulses were monophasic (as shown). In Experiment II, only one channel (left or right) was presented.

Fig. 2 (color online). JNDs as a function of pulse rate obtained from Experiment I for each of the CI listeners. The parameter is the type of ITD, as depicted in Figure 1. Error bars indicate the bootstrap 95\% confidence intervals. Cases where a JND was not determinable at the specified percent correct point are plotted at the top of the figure, marked with ND. For better visual separation of the data points, different conditions are horizontally shifted relative to each other by a small amount. For some conditions the error bars are smaller than the symbols.

Fig. 3 (color online). The mean JNDs of five NH listeners are shown. The left panel plots the JNDs determined using to the $65 \%$ threshold criterion. For comparison, the right panel shows the JNDs using the $80 \%$ criterion. Error bars indicate \pm 1 standard deviation of the mean. Note the different scaling of the ordinates in the two panels. The JND for condition Offset at 400 pps (marked with an asterisk) is based on two listeners for which a JND could be determined. All other conventions are as in Fig. 2. 


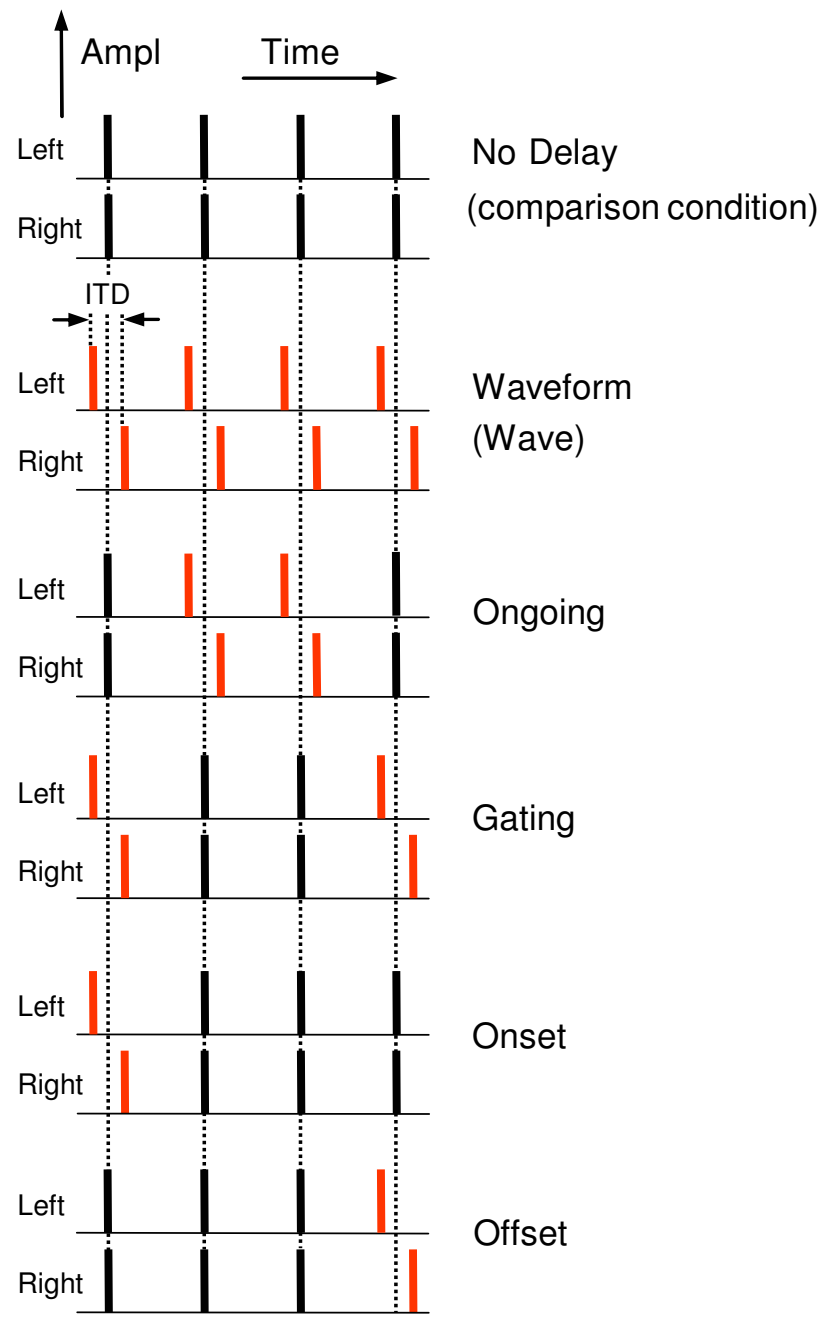

Figure 1 

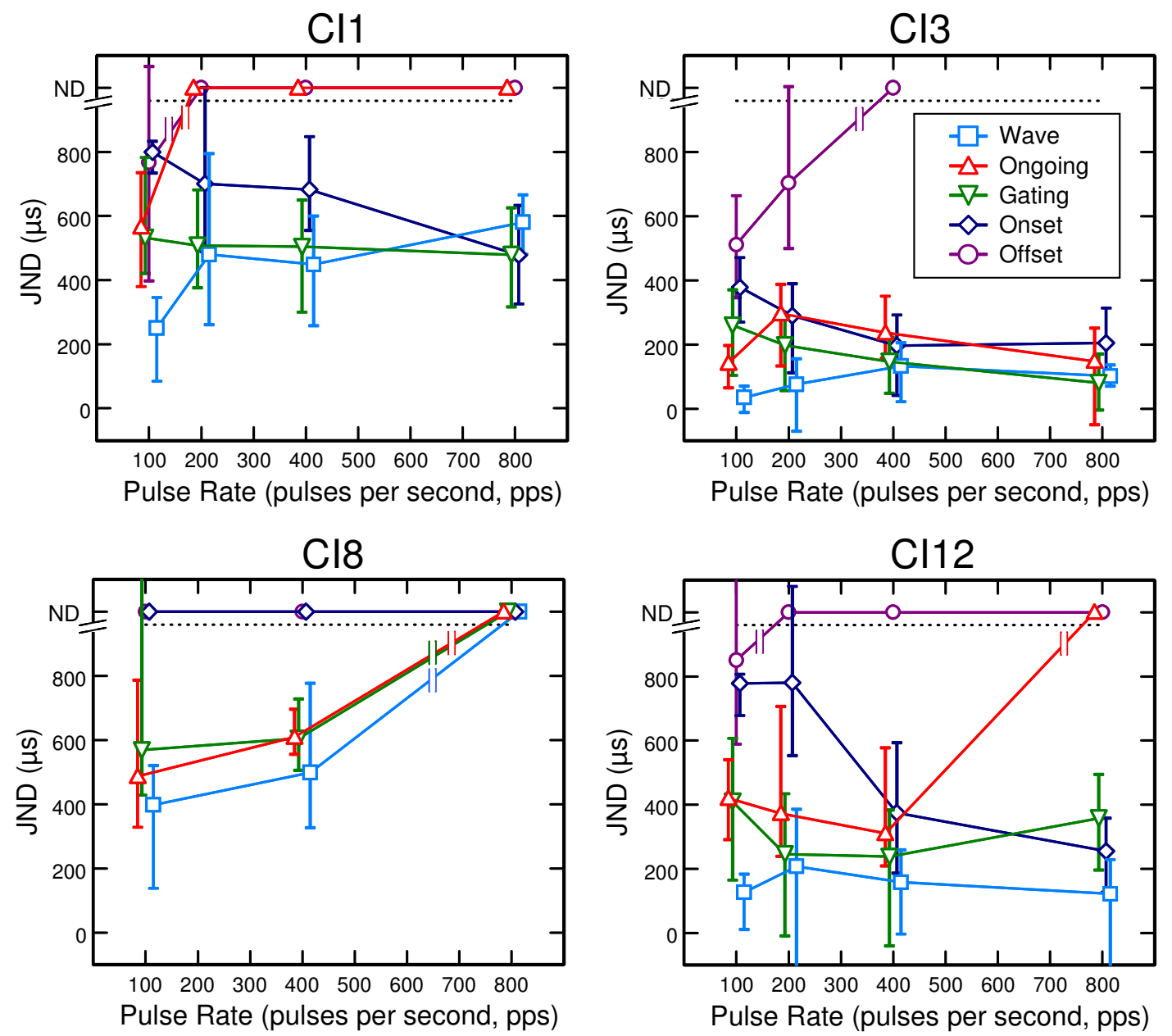

Figure 2 

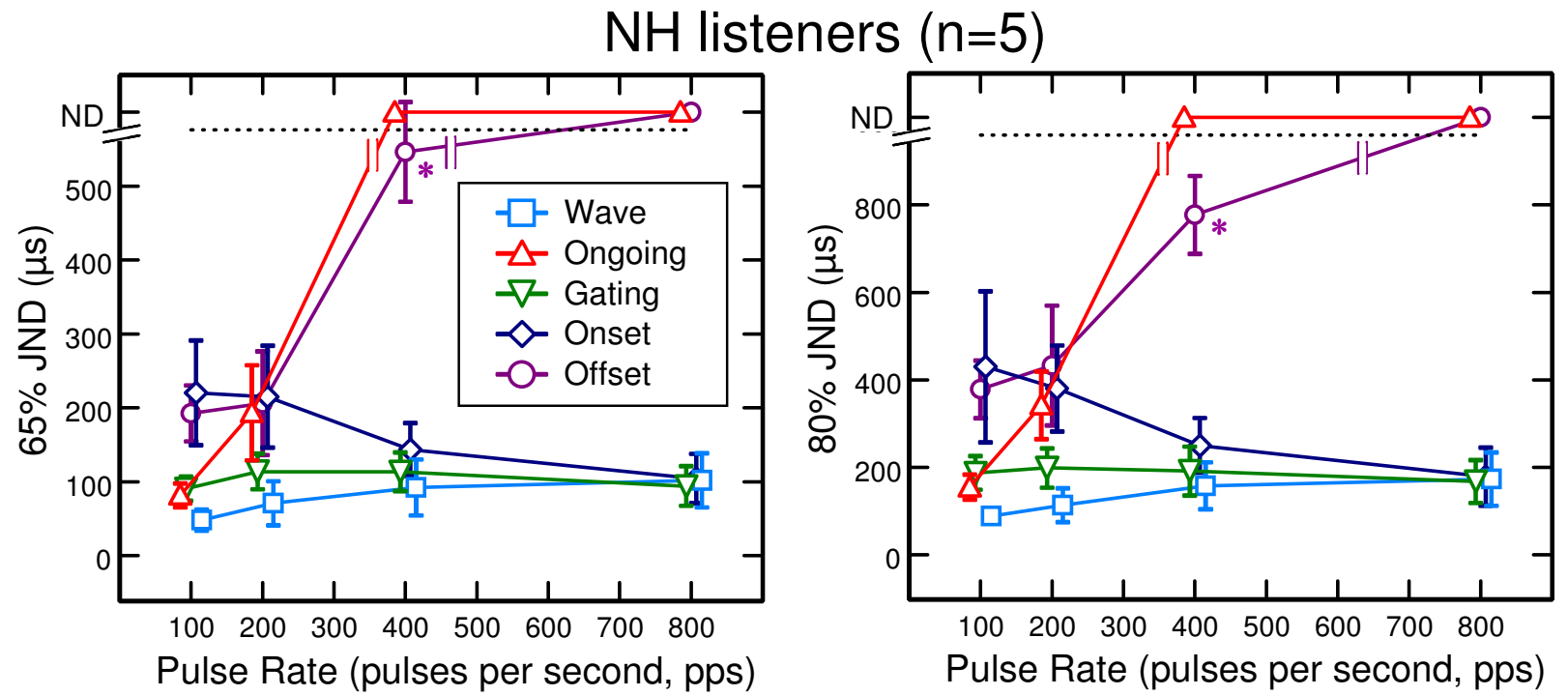

Figure 3 